\title{
Natural history of autoimmune primary ovarian insufficiency in patients with Addison's disease: from normal ovarian function to overt ovarian dysfunction
}

\author{
Annamaria De Bellis"1, Giuseppe Bellastella1,*, Alberto Falorni², Ernesto Aitella3, Mariluce Barrasso1, \\ Maria Ida Maiorino', Elio Bizzarro ${ }^{4}$, Antonio Bellastella', Dario Giugliano ${ }^{1}$ and Katherine Esposito ${ }^{5}$, On the \\ behalf of the Italian Addison Network
}

\begin{abstract}
${ }^{1}$ Endocrinology and Metabolic Diseases Unit, Department of Medical, Surgical, Neurological, Metabolic Sciences and Aging, University of Campania 'L. Vanvitelli', Naples, Italy, ${ }^{2}$ Section of Internal Medicine and Endocrine and Metabolic Sciences, Department of Medicine, University of Perugia, Perugia, Italy, ${ }^{3}$ Department of Clinical and Experimental Medicine, University of Campania 'L. Vanvitelli', Naples, Italy, ${ }^{4}$ Division of Obstetrics and Gynecology, Department of Public Health, School of Medicine and Surgery Department of Public Health 'Federico II' University of Naples, Naples, Italy, ${ }^{5}$ Diabetes Unit, Department of Medical, Surgical, Neurological, Metabolic Sciences and Aging, University of Campania 'L. Vanvitelli', Naples, Italy

*(A De Bellis and G Bellastella contributed equally to this work)
\end{abstract}

Correspondence should be addressed to A De Bellis Email

annamaria.debellis@unina2.it

\begin{abstract}
Context: Women with autoimmune Addison's disease with normal ovulatory cycles but positive for steroid cell antibodies (StCA) have been considered at risk of premature ovarian insufficiency (POI).

Design: Thirty-three women younger than 40 years, with subclinical-clinical autoimmune Addison's disease but with normally ovulatory menses, were followed up for 10 years to evaluate the long-term time-related variations of StCA, ovarian function and follicular reserve. All patients and 27 control women were investigated at the start and every year for the presence and titre of StCA (by indirect immunofluorescence), serum concentrations of anti-Mullerian hormone (AMH) and ovarian function at four consecutive menses every year.

Results: At the start of the study StCA were present in 16 women (group 1), at low/middle titres $(\leq 1: 32)$ in seven of them $(43.8 \%$, group $1 A)$, at high titres $(>1: 32)$ in the remaining nine patients (group $1 B, 56.2 \%)$, while they were absent from 17 patients (group 2). During the follow-up period, all women in group 1A remained StCA-positive at low/middle titres with normal ovulatory menses and normal gonadotrophin and AMH levels, while all patients in group 1B showed a further increase of StCA titres (1:128-1:256) and progressed through three stages of ovarian function. None of the patients in group 2 and controls showed the appearance of StCA or ovarian dysfunction during the follow-up.
\end{abstract}

Conclusions: The presence of StCA at high titres can be considered a good predictive marker of subsequent development of autoimmune POI. To single out the stages of autoimmune POI may allow a timely therapeutic choice in the subclinical and early clinical stages.

\section{Introduction}

The definition of premature ovarian insufficiency (POI) has replaced the old term premature ovarian failure (POF) to emphasise the progressive decline in ovarian function in
두 2017 European Society of Endocrinology Printed in Great Britain women aged younger than 40 years (1). This term indicates a condition characterised by an unpredictable course from normal ovulatory cycles and possible natural pregnancy

Published by Bioscientifica Ltd. 
to a condition of hypergonadotrophic amenorrhoea and infertility. In this time span, normal/high FSH levels and sporadic ovulatory cycles, due to persistence of residual follicular activity, may still be observed (2, 3, $4,5)$. As in other endocrine diseases, some cases of POI fulfill the criteria defining an autoimmune disease, such as lymphomonocytic infiltration of the target gland, presence of serum antibodies against the respective target organs, association with other autoimmune diseases and family history of autoimmune diseases $(6,7)$. Concerning this, a high prevalence of POI has been shown in women with autoimmune diseases such as autoimmune thyroid disease, type 1 diabetes, celiac disease, myasthenia gravis and systemic lupus erythematosus (6). Recently, a particularly high prevalence of association of POI with autoimmune thyroid disease, in the context of autoimmune polyglandular syndrome type 3 (APS-3), has been found by Szlendak-Sauer et al. in 33 of 98 patients (33.7\%) with POI (8). However, autoimmune oophoritis has been unequivocally demonstrated by ovarian biopsy $(2,6)$ almost exclusively in women with POI associated with autoimmune Addison's disease and the presence of serum steroid cell antibodies (StCA-related POI) $(9,10,11$, $12,13)$. In fact, in these cases, histopathological findings show selective lymphomonocytic infiltration around the cells of the growing follicle theca. StCAs were detected by indirect immunofluorescence in cryostat sections of the adrenal gland, ovary, testis and placenta, indicating that the self-antigens recognised by these antibodies are not confined to the ovary, but also expressed in the adrenal cortex and testis $(12,13,14,15,16)$. With regard to ovarian tissue sections, the immunofluorescence pattern of StCA is restricted to the theca cells of the growing follicles. It has been observed that StCA are present in sera of the majority of patients with autoimmune Addison's disease and POI (80-90\%) (12). The finding of antibodies to 17- $\alpha$ hydroxylase (17-OHAb) (17) and to P-450 sidechain cleavage enzyme (P-450sccAb) (18) in some of these sera, suggests that $17-\mathrm{OH}$ and $\mathrm{P}-450$-scc can be considered the main targets of StCA $(13,15)$. However, StCA or $17-\mathrm{OHAb}$ and P-450sccAb may also be detected before the development of POI in some women with autoimmune Addison's disease and with still normal ovulatory cycles (16). Few longitudinal studies have been carried out on the preclinical stage preceding autoimmune POI. In particular, several years ago, some authors investigated longitudinally the immunological and functional pattern of patients with autoimmune Addison's disease, positive for adrenal cortex autoantibodies (ACA), 21-OHAb and StCA but without POI $(11,13,19)$. They found that these patients may progress, during a follow-up period, from a potential stage of autoimmune POI with persisting normal ovulatory menses and normal levels of FSH and $\mathrm{LH}$, directly to a clinically overt stage of POI with development of hypergonadotrophic amenorrhoea. By contrast, results from Nelson $(20,21)$ suggested that the progression from a potential to clinically overt stage of POI is characterised by many more complex intermediate occult and biochemical states in which regular menses, high FSH levels and reduced fertility may be observed. However, at this time, the occurrence of the subclinical phase of autoimmune POI associated with reduced fertility has not been clearly demonstrated, even though the possible occurrence of resumption of ovarian function in patients with POI has been reported (22). With this in mind, and considering that the evaluation of anti-Mullerian hormone (AMH) has been considered a reliable tool to identify patients at risk of developing POI $(23,24,25)$, we planned this longitudinal study, aimed at investigating, in Italian women with autoimmune Addison's disease but with still normally ovulatory cycles, the time-related relationship between StCA appearance and ovarian function associated with assessment of follicular reserve by the evaluation of AMH levels.

\section{Patients and methods}

This is a 10-year prospective observational study including 33 women (aged less than 40 years) with autoimmune Addison's disease, enrolled from 1993 to 2004. In particular, 25 of them showed symptoms and signs of adrenal insufficiency with low basal cortisol and high basal ACTH levels. They belonged to the large cohort of patients, previously diagnosed and included among the Italian Addison network study, a study group consisting of 14 endocrinology centres identified by the Italian Society of Endocrinology for the etiological classification of Addison's disease (26). Moreover, eight women (aged less than 40 years) with subclinical autoimmune Addison's disease, recruited at the Endocrinology Unit of the University of Campania 'Luigi Vanvitelli' among a large cohort of patients with organ-specific autoimmune diseases, such as thyroid autoimmune diseases, type 1 diabetes mellitus, celiac disease, and vitiligo, were also included in the study. They did not show clinical signs and symptoms of Addison's disease but hormonal pattern characteristics of functional adrenal stage 2 of the disease, such as high PRA with normal/low aldosterone levels, normal basal ACTH and cortisol concentrations 
but impaired cortisol response to ACTH stimulation, as previously described (29). To be included into our study, all the patients had to be previously found positive for ACA and 21-OHAb (in the four laboratories of the study group selected for the immunological analysis) but had to present with still regular ovulatory menses, normal body mass index and normal female karyotype. All of them had a family history of autoimmune diseases. Exclusion criteria were the presence of family history of the fragile $\mathrm{X}$ syndrome, POI, hyperprolactinemia, dementia, tremor, ataxia or symptoms and signs likely indicating Parkinson's disease. Patients with clinically overt Addison's disease were under replacement corticosteroid/fludrocortisone therapy $(27,28)$.

Moreover, 27 age (18-27 years) and body massmatched normal women with regular ovulatory menses and lifestyle habits comparable to those of the patients, enrolled among the female students, doctors and endocrinologists attending our department, were also studied as control group. All patients and controls gave their written informed consent to be enrolled into this longitudinal study, which was approved by the Local Institutional Review Board.

\section{Study protocol}

Ovarian function was studied in all patients and in the control group, at the beginning of the study and for four consecutive menses every year until the end of the follow-up (10 years) or at the time of the onset of POI. Antibody evaluation and the serum AMH concentrations were determined in all patients and controls at the start and every year at the occurrence of a menstrual cycle, when occurring, until the end of the study.

\section{Ovarian function evaluation}

In all women LH, FSH (by immunometric assay by monoclonal antibodies) and 17- $\beta$ - oestradiol (by RIA) were measured in the early follicular phase and values were considered normal when the concentration of gonadotrophins was less than $10 \mathrm{mU} / \mathrm{mL}$ and those of $17-\beta$-oestradiol ranged from $50 \mathrm{pg} / \mathrm{mL}$ to $110 \mathrm{pg} / \mathrm{mL}$. Moreover, the finding of progesterone levels (by RIA) $>10 \mathrm{ng} / \mathrm{mL}$ in two serial assays on samples obtained in the mid-luteal phase of the cycle was taken as indicative of ovulation with its consequent luteal phase. We defined normal fertility as ovulation present in all 4 consecutive months, subfertility as ovulation occurring in at least one cycle of the 4 consecutive months, and infertility as ovulation was lacking for all 4 consecutive months.

According to Nelson $(20,21)$ and the most recent recommendations of ESHRE guideline group (5), primary ovarian insufficiency is defined as a loss of ovarian activity before 40 years of age, characterized by menstrual disturbance (amenorrhoea or oligomenorrhoea) for at least 4 months associated with high gonadotrophin and low oestradiol levels.

\section{Antibody evaluation}

ACA and StCA were evaluated in all patients and controls at the start and every year, until the end of follow-up period; by contrast 21-OHAb, 17-OHAb and P450sccAb were determined only at the start of the follow-up period in all patients. ACA and StCA were detected by indirect immunofluorescence on cryostat sections of monkey adrenal, ovary and testis with initial 1:8 serum dilution, as previously described (12). Sera reacting only against the cytoplasm of adrenal cortex cells were considered ACA positive; those reacting against the cytoplasm of adrenal cortex cells, Leydig cells and ovarian theca cells were considered StCA-positive. With regards to the StCA level, we considered arbitrarily a titre of $1: 32$ as a cut-off between low-middle and high titre. Finally, 21-OHAb, 17-OHAb and P450sccAb were measured by radiobinding assay with recombinant human 21-hydroxylase, 17 $\alpha$-hydroxylase and cholesterol side chain cleavage enzyme radiolabelled with ${ }^{35}$, as previously described $(16,28,29)$.

\section{AMH detection}

Serum AMH was measured by ELISA using the AMH/MIS ELISA kit, as previously described (16). The normal values in fertile women ranged from $3 \mathrm{ng} / \mathrm{mL}$ to $6 \mathrm{ng} / \mathrm{mL}$.

Moreover, in all patients and controls, thyroid function and prolactin were also evaluated from year to year. Finally, at the end of the study, all patients with onset of clinical POI underwent a pelvic ultrasound examination (antral follicle count) and Fragile X premutation testing.

\section{Results}

On the basis of the presence or absence of StCA at the start of the study, patients were divided in two groups: group 1, consisting of 16 women (age range 15-25 years) who were StCA positive; group 2, consisting of 17 women (age range 18-26 years) who were StCA negative. 
Table 1 Basal features of the 33 women aged less than 40 years affected by autoimmune Addison's disease with regular ovulatory cycles at the enrolment.

\begin{tabular}{|c|c|c|}
\hline & StCA-positive patients (Group 1) & StCA-negative patients (Group 2) \\
\hline Number of patients & 16 & 17 \\
\hline Age at the start of the study (years) & $15-25$ & $18-26$ \\
\hline Clinically overt Addison's disease, $n(\%)$ & $15(94)$ & $10(59)$ \\
\hline Subclinical Addison's disease, $n(\%)$ & $1(6)$ & $7(41)$ \\
\hline ACA positive, $n(\%)$ & $16(100)$ & $17(100)$ \\
\hline $210 \mathrm{HAb}$ positive, $n(\%)$ & $16(100)$ & $17(100)$ \\
\hline $170 \mathrm{HAb}$ positive, $n(\%)$ & $2(12.5)$ & 0 \\
\hline P450sccAb positive, $n(\%)$ & $2(12.5)$ & 0 \\
\hline Association with other autoimmune disease, $n(\%)$ & $10(62.5)$ & $13(76.5)$ \\
\hline
\end{tabular}

ACA, adrenal cortex antibodies.

The basal characteristics of the patients are illustrated in Table 1 . With regard to the group 1 patients, two showed not only the presence of StCA but also 17-OH and P450 antibodies (12.5\%). For the association with other autoimmune diseases, 10 out of 16 in group 1 (62.5\%) and 13 out of 17 in group 2 (76.5\%) presented coexistence of other autoimmune diseases. In particular, according to the revised classification (30) of APS, complete APS type 1 was observed in one of patients in group 1, incomplete and complete APS type 2 were evident in one and in six, women in group 1, respectively and in seven and three in group 2, respectively; while incomplete APS type 4 was diagnosed in two and in three patients in group 1 and in group 2, respectively. As regards the titre of StCA in patients in group 1, these antibodies at the start were present at low/middle titres (from 1:8 to 1:32) in seven (43.7\%, group 1A) and at high titres ( $>1: 32)$ in nine $(56.2 \%)$, group 1B). Time-related variations of StCA, $\mathrm{LH}, \mathrm{FSH}$ and $\mathrm{AMH}$ in group $1 \mathrm{~A}, 1 \mathrm{~B}$ and group 2 (StcAnegative patients) are depicted in Fig. 1. In particular, the left side of the figure illustrates the variations of patients in group $1 \mathrm{~A}$ and of controls, while the right side of the figure illustrates the variations of patients in group $1 \mathrm{~B}$ and group 2. During the follow-up, all patients in group $1 \mathrm{~A}$ remained StCA-positive at low/middle titres without further increase of the titre (Fig. 1a, left side), normal LH/FSH levels (Fig. 1b and c, left side) and AMH concentrations (Fig. 1d, left side) and without alterations of their menstrual cycles. In contrast, all patients in group $1 \mathrm{~b}$, starting from the fourth year and until the end of the follow-up, showed a further progressive increase of the StCA titres ( $P<0.001$ vs previously observed titres) progressing through three stages of ovarian function (Fig. 1a, b, c, and d, right side, Tables 2 and 3). In particular, Stage 1, characterised by normal ovulation in all four consecutive menses associated with $\mathrm{LH} / \mathrm{FSH}$ and $\mathrm{AMH}$ concentrations in the normal range; Stage 2 (subclinical stage), characterised by an increase in all nine patients of both StCA titre and LH and FSH levels starting from the fourth year of observation $(P<0.001$, vs previous levels) but with still normal ovulatory menses and AMH concentrations; Stage 3a (early clinical stage of POI), characterised by oligomenorrhoea and subfertility (occurrence of at least one ovulation in the four cycles investigated) starting from about the seventh year of observation, associated with persistent high gonadotrophin levels but a significant decrease in $\mathrm{AMH}$ concentrations ( $P<0.001$ vs previous values). In this stage, the presence of residual follicles was evident from trans-vaginal ultrasound in all patients, as well as the absence of the Fragile $X$ premutation, by the appropriate specific testing. Stage $3 b$ (late clinical stage of POI), characterised by clinically overt POI in seven of the nine patients presenting with menstrual disturbance (oligomenorrhoea in three, amenorrhoea in four), further decrease of $\mathrm{AMH}$ concentrations and further increase of FSH and LH levels (in the 8 years in two patients, and at the end of the follow-up in the remaining five). The decreased concentration of $\mathrm{AMH}$ at the beginning of overt POI was particularly remarkable compared with the values detected in the previous phases.

All patients in group 2 (Fig. 1, right side) and normal controls (Fig. 1, left side), negative for StCA at the start, remained StCA-negative throughout the whole follow-up period, without ovarian dysfunction and with $\mathrm{AMH}$ concentrations persistently in the normal range. As result, a significantly higher incidence $(P<0.001)$ of POI was observed in women with autoimmune Addison's disease in group 1 than in those in group 2. As regards the potential of developing ovarian failure (subclinical and clinical POI), we found a positive predictive value of $100 \%$ when the initial titre of StCA was $>1: 32$, while a predictive value was not found when the initial titre was $\leq 1: 32$. Finally, none of patients and controls showed 
A

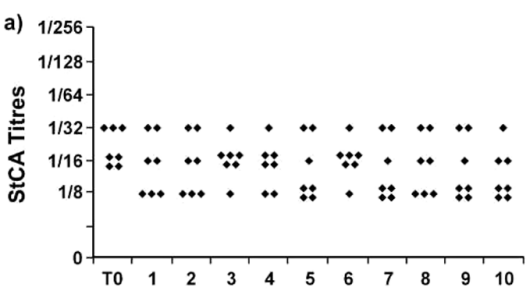

b)

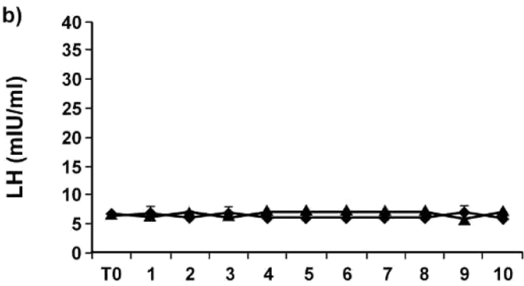

c)
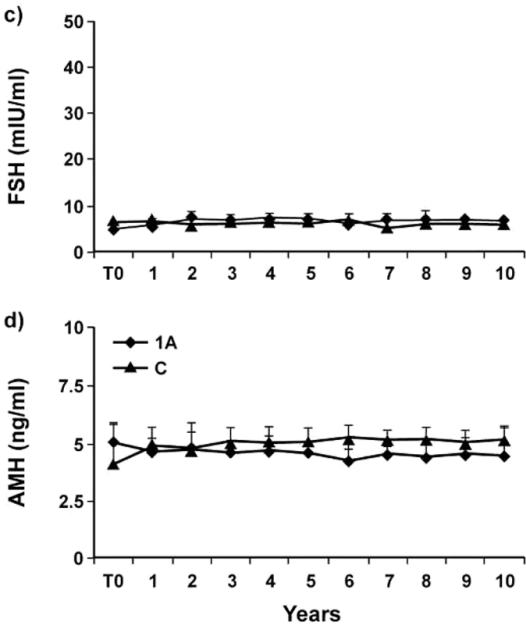

B

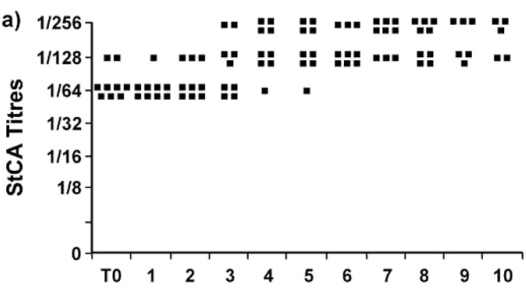

b)
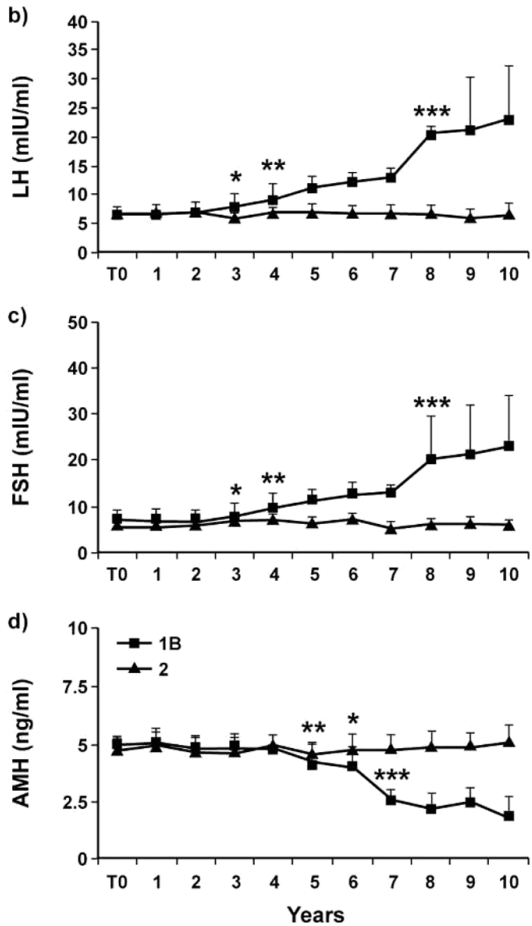

\section{Figure 1}

Time-related variations of StCA, LH, FSH and $\mathrm{AMH}$ during a 10-year follow-up in patients StCA positive at low/middle (group 1A) and high (group 1B) titres, in patients StCA negative at entry (group 2) and in controls $(C)$ : the variations in group $1 \mathrm{~A}$ are illustrated in the left side of the figure compared with those of controls, while those in group 1B are illustrated in the right side of the figure, compared to those of patients in group 2. *from this point, the values significantly differ in the two groups $(P<0.05)$; **from this point the values significantly differ from those at TO (FSH, $P<0.01 ; \mathrm{LH}, P<0.004$; AMH, $P<0.03) * * *$ from this point, the values significantly differ from those at the seventh year (only for $\mathrm{AMH}$ at the sixth year) $(P<0.02)$ and from those at T0 $(P<0.001)$. alterations of thyroid function and of prolactin levels throughout follow-up.

\section{Discussion}

The natural history of autoimmune endocrine diseases usually evolves through three main phases: potential, subclinical and clinically overt dysfunction of the affected endocrine gland. The predictive role of the respective organ-specific antibodies in these diseases seems to depend on their initial titres: in particular, when present at high titre in preclinical phase, they have been considered good predictive markers of subsequent clinical impairment of the respective glands $(15,29,30,31,32,33)$. According to our results, the predictive role of StCA for the future development of POI seems also to depend on the starting titre in patients with autoimmune Addison's disease. In fact, in our patients, the high basal titre with progressive increase during the follow-up was accompanied by a progression from regular ovulatory menses, to subsequent irregular menses and subfertility until a clinically overt POI. Moreover, we have demonstrated for the first time, that in the natural history of autoimmune POI, the potential stage does not progress directly to the clinical stage, as previously described $(11,13,19)$, but through the occurrence of a subclinical stage, characterised by the progressive increase in StCA titres and gonadotrophin concentrations, accompanied by normal ovulatory menses and normal AMH concentrations. Subsequently, the transition to the clinical stage is characterised by a further increase in StCA titre and gonadotrophin levels, and a progressive significant decrease in AMH concentrations, associated with ovarian dysfunction. Consequently, we hypothesise that, as described for other organ-specific antibodies in other autoimmune endocrine diseases, the high basal StCA titre and its subsequent progressive increase should be considered an accurate marker of evolution of the immune process involving the theca of growing follicles, leading to autoimmune oophoritis in the clinical stage of autoimmune POI. However, a possible role for inhibin in discriminating autoimmune oophoritis 
Table 2 Singular behaviour of steroid cell antibodies (StCA titres), FSH levels (IU/mL) and AMH values (ng/mL) of nine at high titre StCA positive patients

\begin{tabular}{|c|c|c|c|c|c|c|c|c|c|c|c|c|c|c|c|}
\hline \multirow[b]{2}{*}{ Patients } & \multicolumn{3}{|c|}{ TO } & \multicolumn{3}{|c|}{ T1 } & \multicolumn{3}{|c|}{ T2 } & \multicolumn{3}{|c|}{ T3 } & \multicolumn{3}{|c|}{ T4 } \\
\hline & FSH & $\mathrm{AMH}$ & STcAb & FSH & $\mathrm{AMH}$ & STcAb & FSH & $\mathrm{AMH}$ & STcAb & $\mathrm{FSH}$ & $\mathrm{AMH}$ & STcAb & FSH & AMH & STcAb \\
\hline 1 & 6.3 & 4.4 & $1 / 64$ & 7.5 & 5 & $1 / 64$ & 6.8 & 4 & $1 / 64$ & 12 & 4.7 & $1 / 128$ & 12.5 & 4 & $1 / 256$ \\
\hline 2 & 6.4 & 4.1 & $1 / 64$ & 6.7 & 4,5 & $1 / 64$ & 6.5 & 4 & $1 / 64$ & 6.9 & 4.1 & $1 / 128$ & 11.9 & 4.2 & $1 / 256$ \\
\hline 3 & 6.7 & 5.4 & $1 / 64$ & 5.8 & 6 & $1 / 64$ & 7.2 & 5.3 & $1 / 64$ & 9.1 & 5.1 & $1 / 256$ & 12 & 4.1 & $1 / 128$ \\
\hline 4 & 5.8 & 4.3 & $1 / 64$ & 6.5 & 4.1 & $1 / 128$ & 6 & 5.1 & $1 / 64$ & 6.8 & 4 & $1 / 128$ & 6 & 4.7 & $1 / 256$ \\
\hline 5 & 6.4 & 5.4 & $1 / 128$ & 7.5 & 5.3 & $1 / 64$ & 11 & 4.1 & $1 / 128$ & 13 & 4 & $1 / 256$ & 13.8 & 4.3 & $1 / 128$ \\
\hline 6 & 6.7 & 6 & $1 / 64$ & 6.8 & 5 & $1 / 64$ & 6 & 5.4 & $1 / 64$ & 6.8 & 6 & $1 / 64$ & 6 & 5.2 & $1 / 128$ \\
\hline 7 & 5.7 & 6 & $1 / 64$ & 5.6 & 5.8 & $1 / 64$ & 6.6 & 5.4 & $1 / 128$ & 5.9 & 5 & $1 / 64$ & 6.1 & 4.4 & $1 / 128$ \\
\hline 8 & 6.8 & 4.5 & $1 / 64$ & 6.6 & 4.3 & $1 / 64$ & 6.7 & 4.5 & $1 / 64$ & 5.5 & 4.6 & $1 / 64$ & 6 & 5.6 & $1 / 64$ \\
\hline 9 & 5.9 & 4.9 & $1 / 128$ & 7.1 & 5 & $1 / 64$ & 5.8 & 4.7 & $1 / 128$ & 7 & 5.2 & $1 / 64$ & 11.5 & 5.4 & $1 / 256$ \\
\hline
\end{tabular}

from other forms of POI has to be taken into account (34). The persistence of normal ovulatory menses in relation to the initial increase of StCA titres can be explained by a possible compensatory role of increased pituitary FSH and LH release, while the further progressive increase of these antibodies during the follow up could reflect a time-related partial destruction of theca cells, with consequent further increases in FSH/LH concentration and still not irreversible ovarian dysfunction (early clinical stage), characterised by the occurrence of oligomenorrhoea associated with subfertility in some patients. Thus, our results indicate that the appearance of clinical autoimmune POI may not only be characterised by amenorrhoea, but also by the presence of irregular menses with further reduction (but still possible persistence) of fertility and that the progression from a potential to clinical stage of POI in our patients was strictly correlated with the time-related variations of StCA titre. For this reason, we suggest that the transition from the subclinical to the early clinical stage of autoimmune POI corresponds to the occult-biochemical states of POI previously described (35). Only when the prolonged autoimmune attack, with further StCA titre increase, irreversibly damages the follicular reserve of the ovary (late clinical stage) do AMH concentrations dramatically decrease, accompanied by stable hypergonadotrophic oligomenorrhoea/amenorrhoea and infertility. Previous studies have recommended the screening for 21-OHAb, or alternatively ACA, in POI associated with suspected autoimmune disorders and in POI of unknown causes (5). However, considering the occurrence of subclinical or clinical POI in our StCA-positive patients, but not in those StCA-negative (even if positive for ACA and 21-OH $\mathrm{Ab}$ ), we suggest on the one hand that the prevalence of autoimmune POI in patients with autoimmune Addison's disease positive for StCA could be higher than that so far reported, and on the other that only StCA, but not ACA and 21-OHAb, may be considered a predictive marker of this ovarian dysfunction, particularly when persistent and present at high titres. Thus, an immunofluorescence screening for StCA simultaneously on the testis, ovary and adrenal gland on the same glass slide, avoiding searching for ACA or 21-OHAb, may be considered an appropriate diagnostic tool, also reducing the cost/benefit ratio. However, the relationship between the different adrenal antibodies and StCA in patients with Addison's and POI is still being discussed. In our previous cross-sectional and longitudinal studies, a strong correspondence between ACA and 21-OHAb was observed both in subclinical and clinical Addison's disease (29); however, the presence of $17 \mathrm{OHAb}$ and P450sccAb was not always associated with the presence of StCA in the sera of patients with Addison's disease and POI, at least in the first observations $(12,13)$. In fact, some sera positive for 17OHAb and P450scc $\mathrm{Ab}$ at low titres were negative for StCA, even though a subsequent seroconversion for StCA was observed in all cases. Conversely, other sera found positive for StCA appeared negative for $17 \mathrm{OHAb}$ and P450sccAb.

Brozzetti et al., testing the sera of 112 females with autoimmune Addison's disease without POI (belonging to this multicentre study and thus also including our patients), showed that only $10 \%$ and $14 \%$ were positive for $17 \mathrm{OHAb}$ and $\mathrm{P} 450 \mathrm{scc} \mathrm{Ab}$, respectively (27). The presence of $17 \mathrm{OHAb}$ and P450sccAb only in two (12\%) of a cohort of StCA-positive patients suggests that a serum reactivity to as yet unidentified autoantigens (different from $17 \alpha$ $\mathrm{OH}$ or P450scc) could be responsible for a positive immunofluorescence signal (13). Thus, even if StCA could appear to be highly specific for the development of POI, further comparative studies on the longitudinal behaviour of 17OHAb, P450sccAb and StCA in a greater cohort of patients are needed to clarify these aspects further and confirm the specificity of these antibodies. 


\begin{tabular}{|c|c|c|c|c|c|c|c|c|}
\hline \multicolumn{3}{|c|}{ T5 } & \multicolumn{3}{|c|}{ T6 } & \multicolumn{3}{|c|}{ T7 } \\
\hline FSH & $\mathrm{AMH}$ & STcAb & FSH & $\mathrm{AMH}$ & STcAb & FSH & $\mathrm{AMH}$ & STcAb \\
\hline 13 & 2.8 & $1 / 128$ & 14 & 2.5 & $1 / 256$ & 17 & 2.1 & $1 / 256$ \\
\hline 12.7 & 4 & $1 / 128$ & 12.3 & 5.4 & $1 / 256$ & 13 & 3.1 & $1 / 256$ \\
\hline 13.9 & 3.1 & $1 / 256$ & 13.4 & 3 & $1 / 128$ & 14 & 3 & $1 / 256$ \\
\hline 8.7 & 5.2 & $1 / 256$ & 12.7 & 3.8 & $1 / 128$ & 14 & 2.8 & $1 / 128$ \\
\hline 14.8 & 3.8 & $1 / 256$ & 14 & 3.7 & $1 / 128$ & 16 & 2.3 & $1 / 128$ \\
\hline 7.8 & 4.1 & $1 / 128$ & 9.9 & 4 & $1 / 128$ & 12 & 3 & $1 / 256$ \\
\hline 6.5 & 4.1 & $1 / 64$ & 11.8 & 4.4 & $1 / 128$ & 13 & 3 & $1 / 256$ \\
\hline 7 & 5.2 & $1 / 128$ & 11.6 & 4.7 & $1 / 128$ & 11.6 & 4.7 & $1 / 128$ \\
\hline 14 & 4.8 & $1 / 256$ & 12.8 & 3.1 & $1 / 256$ & 14.2 & 3 & $1 / 256$ \\
\hline
\end{tabular}

\begin{tabular}{|c|c|c|}
\hline \multicolumn{3}{|c|}{ T8 } \\
\hline FSH & AMH & STcAb \\
\hline 26 & 1.5 & $1 / 128$ \\
\hline 14 & 3 & $1 / 256$ \\
\hline 38 & 2 & $1 / 256$ \\
\hline 13.4 & 2,2 & $1 / 256$ \\
\hline 29 & 1.70 & $1 / 128$ \\
\hline 15.5 & 3 & $1 / 256$ \\
\hline 13.8 & 2.7 & $1 / 256$ \\
\hline 12 & 3.1 & $1 / 128$ \\
\hline 23 & 2.1 & $1 / 128$ \\
\hline
\end{tabular}

\begin{tabular}{cccc}
\hline \multicolumn{3}{c}{ T9 } \\
\cline { 1 - 2 } FSH & & AMH & STcAb \\
\cline { 1 - 1 }- & $\frac{-}{29.8}$ & 2.1 & $1 / 256$ \\
- & - & - \\
18.8 & 3.1 & $1 / 256$ \\
- & - & - \\
15.5 & 3 & $1 / 128$ \\
13 & 2 & $1 / 128$ \\
20 & 2.5 & $1 / 128$ \\
33 & 2 & $1 / 256$ \\
\hline
\end{tabular}

\begin{tabular}{ccccc}
\hline \multicolumn{3}{c}{ T10 } \\
\cline { 1 - 1 } FSH & & AMH & & STcAb \\
\cline { 1 - 1 }$\frac{-}{39}$ & & - & & - \\
-4 & & $1 / 256$ \\
- & - & & - \\
28 & & 2.1 & & $1 / 256$ \\
- & - & & - \\
15 & 2 & & $1 / 128$ \\
13 & 2 & & $1 / 128$ \\
26 & 1.7 & & $1 / 256$ \\
& &
\end{tabular}

Patient developing overt clinical POI are marked bold.

This could be an advisable choice, not only in women with POI of unknown origin or with a suspected autoimmune disorder, but also in those with an individual or familial history of autoimmunity without ovarian dysfunction but at risk of future POI.

A final but equally interesting message from our longitudinal study emerges from the evaluation of the time-related variations of AMH concentrations, since AMH is considered the best hormonal marker for estimating the ovarian follicle pool and for identifying patients at risk of developing POI $(23,24,25,26,36)$. In a recent Italian Addison network study, La Marca et al. found normal serum AMH concentration in two-thirds of women recently diagnosed with StCA-related POI $(16,33)$ providing the first demonstration of the existence of a subgroup of women with autoimmune POI but with a preserved ovarian follicle pool. The authors suggested that future studies on the dynamic variations of serum AMH concentrations in women with autoimmune Addison's disease at risk of StCA-related POI could provide important information on the rate of loss of follicle reserve.

The longitudinal changes in AMH concentration in our patients with autoimmune Addison's disease, and its relationship with the time-related variations of StCA and ovarian function showed that, when StCA titres increased, oligomenorrhoea associated with high LH/FSH levels and subfertility appeared, and AMH levels were still detectable but significantly lower than those previously found when normal ovulatory menses were still present. Conversely, when clinically overt POI occurred, AMH levels, even if still detectable in some cases, showed a further decrease with respect to those found in the subclinical stage. In this connection, we suggest that the AMH assay may be particularly useful in subclinical (stage 2) and early clinical phase (stage 3a), because the finding of decreased but still detectable values may indicate the persistence of a residual follicular pool. Thus, this staging is particularly relevant for affected women wishing to conceive, because the longitudinal changes in AMH values seems to indicate that a residual follicular pool may persist until the early clinical stage of autoimmune POI, when the autoimmune process has not completely destroyed the theca and granulosa cells, and POI could be still reversible (22), likely allowing natural or assisted pregnancies (37, 38). Moreover, since further important comorbidities are usually associated with clinically overt POI, such as cognitive dysfunction and psychological distress, autoimmune diseases, urogenital atrophy, osteoporosis and cardiovascular diseases $(5,39)$, the staging of POI could also be useful, with timely oestrogen replacement

Table 3 Natural history of autoimmune premature ovarian insufficiency (POI) in patients with Addison's disease positive for steroid cell antibodies (StCA) at high titre.

\begin{tabular}{|c|c|c|c|c|c|}
\hline Stage & StCA titre & Menstrual cycles & FSH and LH levels & AMH values & Fertility \\
\hline 1 & $>1 / 32$ & Regular & Normal & Normal & Normal \\
\hline 2 Subclinical stage & Increased & Regular & Increase & Normal & Normal \\
\hline 3a Early clinical stage & Further increased & $\begin{array}{l}\text { Menstrual disturbances } \\
\text { (oligomenorrhoea) }\end{array}$ & Increase & Decrease & Subfertility \\
\hline 3b Late clinical stage & Further increased & $\begin{array}{l}\text { Menstrual disturbances } \\
\text { (oligomenorrhoea/ } \\
\text { amenorrhoea) }\end{array}$ & Further increase (>25IU/mL) & Further decrease & Infertility \\
\hline
\end{tabular}


therapy, in the prevention or at least minimization of the occurrence of these severe comorbidities, and a possible or at least partial, recovery of ovarian function, as demonstrated for functional recovery occurring in patients with central autoimmune diabetes insipidus and in patients with autoimmune Addison's disease, treated precociously with desmopressin (31) and glucocorticoids (40), respectively. This should also be in agreement with the recent ESHRE guidelines on the management of women with POI (5), recommending oestrogen replacement therapy with early initiation to prevent in particular osteoporosis and cardiovascular diseases, which are frequently the consequence of POI.

The assumptions derived from our results may not be generalised, because they regard only POI affecting women with Addison's disease, in whom the autoimmune adrenal impairment could play a confounding role. Moreover, a further limitation of our study is the small number of patients, especially in $1 \mathrm{~A}$ and $1 \mathrm{~B}$ subgroups, also caused by the withdrawal of some patients during the prolonged follow-up. Future studies have to be planned and performed on a larger group of women in subclinical and early clinical stage of POI also without Addison's disease, aimed at confirming the staging of the disease and the effects of the suggested therapeutic option on their fertility, and also on the occurrence and the progression of associated comorbidities.

Declaration of interest

The authors have nothing to disclose.

Funding

This research did not receive any specific grant from any funding agency in the public, commercial or not-for-profit sector.

\section{Acknowledgements}

In addition to the authors, the following members of the Italian Addison Network contributed to the collection of data and blood samples from patients with autoimmune Addison disease: B Ambrosi, G Angeletti, G Arnaldi, E Arvat, G Basta, P Beck-Peccoz, C Betterle, A Bizzarro, M Boscaro, F Cavagnini, F Calcinaro, R Celleno, C Dal Pra, F Dotta, E Ghigo, L Iorio, S Laureti, F Loré, M Mannelli, F Mantero, F Pecori Giraldi, F Santeusanio, M Terzolo, C Tiberti, P Toja, M Torlontano, V Toscano, $\mathrm{V}$ Trischitta, R Zanchetta. Moreover, the authors wish to thank Dr Trevor G Cooper, Hong Kong SAR, PR China, for his precious suggestions and advices in editing the paper.

\section{References}

1 Welt CK. Primary ovarian insufficiency: a more accurate term for premature ovarian failure. Clinical Endocrinology 200868 499-509. (doi:10.1111/j.1365-2265.2007.03073.x)
2 Bannatyne P, Russell P \& Shearman RP. Autoimmune oophoritis: a clinicopathologic assessment of 12 cases. International Journal of Gynecological Pathology 19909 191-207. (doi:10.1097/00004347199007000-00001)

3 Cox L \& Liu JH. Primary ovarian insufficiency: an update. International Journal of Women's Health 20146 235-243. (doi:10.2147/ IJWH.S37636)

4 Fenton AJ. Premature ovarian insufficiency: pathogenesis and management. Journal of Mid-life Health 20156 147-153. (doi:10.4103/0976-7800.172292)

5 ESHRE Guideline Group on POI, Webber L, Davies M, Anderson R, Bartlett J, Braat D, Cartwright B, Cifkova R, de Muinck Keizer-Schrama S, Hogervorst E, et al. ESHRE guideline: management of women with premature ovarian insufficiency. Human Reproduction 201631 926-937. (doi:10.1093/humrep/dew027)

6 Hoek A, Schoemaker J \& Drexhage HA. Premature ovarian failure and ovarian autoimmunity. Endocrine Reviews 199718 107-134. (doi:10.1210/er.18.1.107)

7 Rose NR \& Bona C. Defining criteria for autoimmune diseases (Witebsky's postulates revisited). Immunology Today 199314 426-430. (doi:10.1016/0167-5699(93)90244-F)

8 Szlendak-Sauer K, Jakubik D, Kunicki M Skorska J \& Smolarczyk R. Autoimmune polyglandular syndrome type 3 (APS-3) among patients with premature ovarian insufficiency (POI). European Journal of Obstetrics and Gynecology and Reproductive Biology 2016203 61-65. (doi:10.1016/j.ejogrb.2016.05.023)

9 Forges T, Monnier-Barbarino P, Faure GC \& Béné MC. Autoimmunity and antigenic targets in ovarian pathology. Human Reproduction Update 200410 163-175. (doi:10.1093/ humupd/dmh014)

10 Irvine WJ, Chan MM, Scarth L, Kolb FO, Hartog M, Bayliss RI \& Drury MI. Immunological aspects of premature ovarian failure associated with idiopathic Addison's disease. Lancet 19682 883-887. (doi:10.1016/S0140-6736(68)91053-2)

11 Betterle C \& Volpato M. Adrenal and ovarian autoimmunity. European Journal of Endocrinology 1998138 16-25. (doi:10.1530/ eje.0.1380016)

12 Falorni A, Laureti S, Candeloro P, Perrino S, Coronella C, Bizzarro A, Bellastella A, Santeusanio F \& De Bellis A. Steroid-cell autoantibodies are preferentially expressed in women with premature ovarian failure who have adrenal autoimmunity. Fertility and Sterility $2002 \mathbf{7 8}$ 270-279. (doi:10.1016/S0015-0282(02)03205-3)

13 Reato G, Morlin L, Chen S, Furmaniak J, Smith BR, Masiero S, Albergoni MP, Cervato S, Zanchetta R \& Betterle C. Premature ovarian failure in patients with autoimmune Addison's disease: clinical, genetic, and immunological evaluation. Journal of Clinical Endocrinology and Metabolism 201196 1255-1261. (doi:10.1210/ jc.2011-0414)

14 Ahonen P, Miettinen A \& Perheentupa J. Adrenal and steroidal cell antibodies in patients with autoimmune polyglandular disease type I and risk of adrenocortical and ovarian failure. Journal of Clinical Endocrinology and Metabolism 198764 494-500. (doi:10.1210/jcem-643-494)

15 Betterle C, Dal Pra C, Mantero F \& Zanchetta R. Autoimmune adrenal insufficiency and autoimmune polyendocrine syndromes: autoantibodies, autoantigens, and their applicability in diagnosis and disease prediction. Endocrine Reviews 200223 327-364. (doi:10.1210/ edrv.23.3.0466)

16 La Marca A, Marzotti S, Brozzetti A, Stabile G, Artenisio AC, Bini V, Giordano R, De Bellis A, Volpe A, Falorni A, et al. Primary ovarian insufficiency due to steroidogenic cell autoimmunity is associated with a preserved pool of functioning follicles. Journal of Clinical Endocrinology and Metabolism 200994 3816-3823. (doi:10.1210/ jc.2009-0817)

17 Krohn K, Uibo R, Aavik E, Peterson P \& Savilahti K. Identification by molecular cloning of an autoantigen associated with Addison's 
disease as steroid 17 alpha-hydroxylase. Lancet 1992339 770-773. (doi:10.1016/0140-6736(92)91894-E)

18 Winqvist O, Gustafsson J, Rorsman F, Karlsson FA \& Kämpe O. Two different cytochrome P450 enzymes are the adrenal antigens in autoimmune polyendocrine syndrome type I and Addison's disease. Journal of Clinical Investigation 196892 2377-2385. (doi:10.1172/ JCI116843)

19 Betterle C, Rossi A, Dalla Pria S, Artifoni A, Pedini B, Gavasso S \& Caretto A. Premature ovarian failure: autoimmunity and natural history. Clinical Endocrinology 199339 35-43. (doi:10.1111/j.1365-2265.1993.tb01748.x)

20 Nelson LM. Clinical practice. Primary ovarian insufficiency. New England Journal of Medicine 2009360 606-614. (doi:10.1056/ NEJMcp0808697)

21 Nelson LM. One world, one woman; a transformational leader's approach to primary ovarian insufficiency. Menopause $2011 \mathbf{1 8}$ 480-487. (doi:10.1097/gme.0b013e318213f250)

22 Bachelot A, Nicolas C, Bidet M, Dulon J, Leban M, Golmard JL, Polak M \& Touraine P. Long-term outcome of ovarian function in women with intermittent premature ovarian insufficiency. Clinical Endocrinology 201786 223-228. (doi:10.1111/cen.13105)

23 La Marca A, Sighinolfi G, Giulini S, Traglia M, Argento C, Sala C, Masciullo C, Volpe A \& Toniolo D. Normal serum concentrations of anti-Müllerian hormone in women with regular menstrual cycles. Reproduction BioMedicine Online 201021 463-469. (doi:10.1016/j. rbmo.2010.05.009)

24 Saglam F, Onal ED, Ersoy R, Koca C, Ergin M, Erel O \& Cakir B. Anti-Müllerian hormone as a marker of premature ovarian aging in autoimmune thyroid disease. Gynecological Endocrinology 201531 165-168. (doi:10.3109/09513590.2014.973391)

25 Nelson SM, Yates RW \& Fleming R. Serum anti-Müllerian hormone and FSH: prediction of live birth and extremes of response in stimulated cycles - implications for individualization of therapy. Human Reproduction 200722 2414-2421. (doi:10.1093/humrep/dem204)

26 Falorni A, Laureti S, De Bellis A, Zanchetta R, Tiberti C, Arnaldi G, Bini V, Beck-Peccoz P, Bizzarro A, Dotta F, et al. Italian addison network study: update of diagnostic criteria for the etiological classification of primary adrenal insufficiency. Journal of Clinical Endocrinology and Metabolism 200489 1598-1604. (doi:10.1210/ jc.2003-030954)

27 Brozzetti A, Alimohammadi M, Morelli S, Minarelli V, Hallgren Å, Giordano R, De Bellis A, Perniola R, Kämpe O, Falorni A, et al. Autoantibody response against NALP5/MATER in primary ovarian insufficiency and in autoimmune Addison's disease. Journal of Clinical Endocrinology and Metabolism 2015100 1941-1948. (doi:10.1210/ jc.2014-3571)

28 Brozzetti A, Marzotti S, La Torre D, Bacosi ML, Morelli S, Bini V, Ambrosi B, Giordano R, Perniola R, De Bellis A, et al. Autoantibody responses in autoimmune ovarian insufficiency and in Addison's disease are IgG1 dominated and suggest a predominant, but not exclusive, Th1 type of response. European Journal of Endocrinology 2010 163 309-317. (doi:10.1530/eje-10-0257)

29 Laureti S, De Bellis A, Muccitelli VI, Calcinaro F, Bizzarro A, Rossi R, Bellastella A, Santeusanio F \& Falorni A. Levels of adrenocortical autoantibodies correlate with the degree of adrenal dysfunction in subjects with preclinical Addison's disease. Journal of Clinical
Endocrinology and Metabolism $1998 \mathbf{8 3}$ 3507-3511. (doi:10.1210/ jc.83.10.3507)

30 Coco G, Dal Pra C, Presotto F, Albergoni MP, Canova C, Pedini B, Zanchetta R, Chen S, Furmaniak J, Rees Smith B, et al. Estimated risk for developing autoimmune Addison's disease in patients with adrenal cortex autoantibodies. Journal of Clinical Endocrinology and Metabolism 200691 1637-1645. (doi:10.1210/jc.2005-0860)

31 De Bellis A, Colao A, Di Salle F, Muccitelli VI, Iorio S, Perrino S, Pivonello R, Coronella C, Bizzarro A, Lombardi G, et al. A longitudinal study of vasopressin cell antibodies, posterior pituitary function, and magnetic resonance imaging evaluations in subclinical autoimmune central diabetes insipidus. Journal of Clinical Endocrinology and Metabolism 199984 3047-3051. (doi:10.1210/jcem.84.9.5945)

32 Bellastella G, Rotondi M, Pane E, Dello Iacovo A, Pirali B, Dalla Mora L, Falorni A, Sinisi AA, Bizzarro A, Colao A, et al. Predictive role of the immunostaining pattern of immunofluorescence and the titers of antipituitary antibodies at presentation for the occurrence of autoimmune hypopituitarism in patients with autoimmune polyendocrine syndromes over a five-year follow-up. Journal of Clinical Endocrinology and Metabolism 201095 3750-3757. (doi:10.1210/jc.2010-0551)

33 La Marca A, Brozzetti A, Sighinolfi G, Marzotti S, Volpe A \& Falorni A. Primary ovarian insufficiency: autoimmune causes. Current Opinion in Obstetrics and Gynecology 201022 277-282. (doi:10.1097/ GCO.0b013e32833b6c70)

34 Tsigkou A, Marzotti S, Borges L, Brozzetti A, Reis F, Candeloro P, Luisa Bacosi M, Bini V, Petraglia F \& Falorni A. High serum inhibin concentration discriminates autoimmune oophoritis from other forms of primary ovarian insufficiency. Journal of Clinical Endocrinology and Metabolism 200893 1263-1269. (doi:10.1210/ jc.2007-1675)

35 Cameron IT, O'Shea FC, Rolland JM, Hughes EG, de Kretser DM \& Healy DL. Occult ovarian failure: a syndrome of infertility, regular menses, and elevated follicle-stimulating hormone concentrations. Journal of Clinical Endocrinology and Metabolism 198867 1190-1194. (doi:10.1210/jcem-67-6-1190)

36 La Marca A \& Volpe A. Anti-Müllerian hormone (AMH) in female reproduction: is measurement of circulating $\mathrm{AMH}$ a useful tool? Clinical Endocrinology 200664 603-610. (doi:10.1111/j.13652265.2006.02533.x)

37 Ben-Nagi J \& Panay N. Premature ovarian insufficiency: how to improve reproductive outcome? Climacteric 201417 242-246. (doi:10.3109/13697137.2013.860115)

38 Kawamura K, Kawamura N \& Hsueh AJ. Activation of dormant follicles: a new treatment for premature ovarian failure? Current Opinion in Obstetrics and Gynecology 201628 217-222. (doi:10.1097/ GCO.0000000000000268)

39 Podfigurna-Stopa A, Czyzyk A, Grymowicz M, Smolarczyk R, Katulski K, Czajkowski K \& Meczekalski B. Premature ovarian insufficiency: the context of long-term effects. Journal of Endocrinological Investigation 201639 983-990. (doi:10.1007/s40618-016-0467-z)

40 De Bellis A, Bizzarro A, Rossi R, Paglionico VA, Criscuolo T, Lombardi $\mathrm{G} \&$ Bellastella A. Remission of subclinical adrenocortical failure in subjects with adrenal autotoantibodies. Journal of Clinical Endocrinology and Metabolism 199376 1002-1007. (doi:10.1210/ jc.76.4.1002)

Received 21 February 2017

Revised version received 14 July 2017

Accepted 21 July 2017 